\title{
Fault location system with injection scheme based on mobile phone remote control in distribution network
}

\author{
Lige $\mathrm{He}^{1, \mathrm{a}}$, Yongqiang Che ${ }^{2, \mathrm{~b}}$, Yulin $\mathrm{Qi}^{2, \mathrm{c}}, \mathrm{Xi} \mathrm{Lu}^{2, \mathrm{~d}}$ \\ ${ }^{1}$ State Grid Hebei Electric Power Company, Zhuozhou Power Supply Company, Zhuozhou 072750, \\ China; \\ ${ }^{2}$ School of Electrical and Electronic Engineering, North China Electric Power University, Baoding \\ 071000, China. \\ azzsjk@sina.com, b15712522507@163.com, 'cqiyulin589@163.com, d18331123700@163.com
}

Keywords: distribution network, fault location, signal source, Android application, cloud server

\begin{abstract}
The signal source based on signal injection scheme which is the main equipment of fault location in distribution network injects AC constant current signal to fault lines. However, its constant current value can't be changed according to characteristics of lines. This paper proposes a high power signal source which can be controlled remotely through an Android mobile phone application. Firstly, the signal source has remote data sent-receive ability through GPRS module. Then data forwarding is realized by cloud server which has a fixed IP. Finally, data exchange between mobile client application and signal source GPRS module is realized by the connection to the cloud server. This achieves that Android client application can remote control the high power signal source for fault location in distribution network.
\end{abstract}

\section{Introduction}

Compared to the transmission network, the fault rate in medium voltage distribution network is much higher relatively ${ }^{[1]}$. In distribution network, single-phase-earth fault that is normal occurrence is also called neutral point ineffectively grounding fault ${ }^{[2]}$. According to statistics, single-phase-earth fault accounts for about $80 \%$ of the total fault in distribution network. It even reaches $90 \%$ if transient fault is considered ${ }^{[3]}$, so it is a main fault in distribution network. Therefore, fault location in distribution network has always been important to power system. It became the hot topics in the study of people, and the impedance scheme, the traveling wave scheme, the signal injection scheme and some related fault location schemes are summarized and developed through constant efforts and exploration by scientific research personnel ${ }^{[4]}$.Some improvements are made on the basis of those schemes ${ }^{[5]}$, and GPRS technology is also used in this field ${ }^{[6]}$.Significant achievements are made for single-phase-earth fault location in distribution network.

On the current, most of signal source with injection scheme injects constant signal to the line. However, staff can't control the signal source remotely after the signal is injected into lines when it works stably. The staff in the process of patrol often needs to control the signal source and adjusts the injected signal. For example: when the length of the line beyond a certain range, it will be difficult to detect the signal; in some special cases such as line tower is too high, and it leads to transmission lines are too far away from ground to detect the signal for stuff. In the two situations above, staff needs to control the signal source remotely. Otherwise, it will bring great inconvenience to fault location. Thus, it can be seen that the signal source remote controllability is very necessary for rapid and accurate fault location.

\section{Overall Design of Fault Location System}

In addition to the signal source, this fault location system also includes three parts: mobile client with Android applications, cloud server with a fixed IP, a handheld detector which can detect the fault point. The above four parts use cooperatively to ensure the accuracy and quickness of fault location. 
Signal source is equivalent to a constant current source. After the fault occurs, system will inject signal to the fault phase from the signal source. When signal source works stably, staff will hold a detector and take an Android phone with corresponding client application to find the fault point along the line. In addition, if the signal source is turned on, it will connect to cloud server with fixed IP automatically by GPRS module, and mobile client application will also connect to the cloud server. After the connection, data forwarding between them is realized. They can communicate with each other to achieve that signal source is controlled by the phone remotely.

Staff in the process of patrol with handheld detector can real-time monitor the condition of signal source through the mobile client application. The current of the injected signal also can be adjusted according to need. This solves the problem that signal is too weak or strong or the interference is too serious to locate fault point effectively. Stuff can also find it in time when the work condition of signal source is not stable, and then it can be adjusted. This avoids the misjudgment problem effectively. Fault location principle is shown in Figure 1.

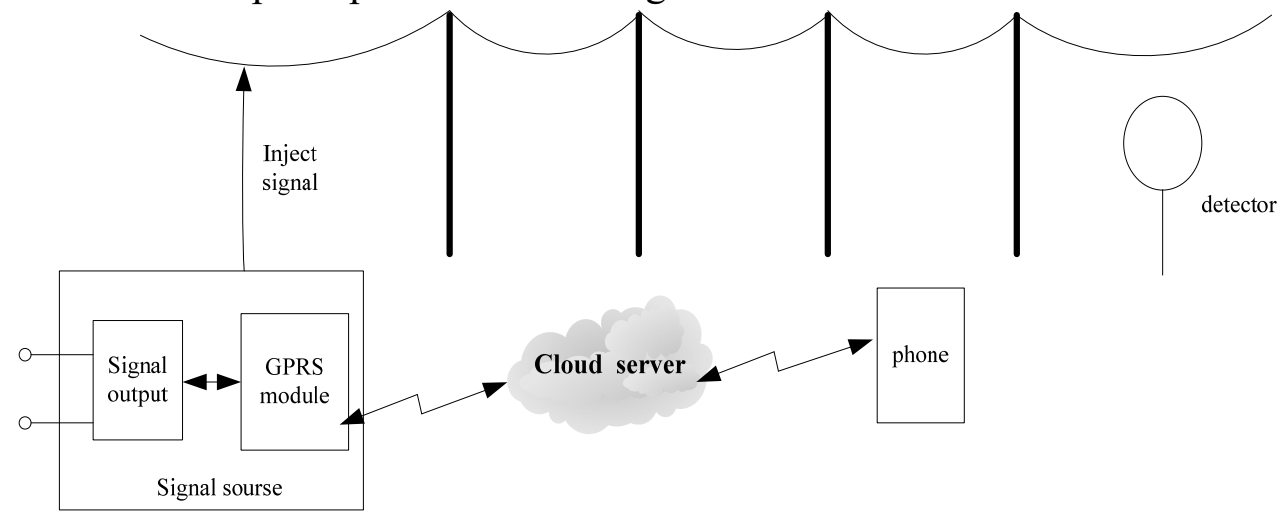

Fig. 1 The principle diagram of the fault location

\section{Design of Signal Source for Fault Location}

Working Principle of Signal Source for Fault Location. Signal source is powered by $220 \mathrm{~V}$ AC. After the system power supply is stable, signal source will inject $150 \mathrm{~mA}$ AC current whose frequency is $60 \mathrm{~Hz}$ to the fault phase automatically. The output will be stable in current of $150 \mathrm{~mA}$, $60 \mathrm{~Hz}$. Signal source is mainly divided into the following three parts: current output module, control module and GPRS module.

(1) Current output module: after the signal source is turned on, current output module will inject signal to the fault phase. The current is controlled by control module.

(2) Control module: the module uses C8051F310 chip, uses PWM technology and the way of full bridge circuit to form rectifier circuit. Control module can receive the external control commands from buttons and GPRS module through I/O port, and then control the current output module. The A/D sampling values of signal will be displayed in the touch screen, at the same time, the information will be sent to the GPRS module.

(3) GPRS module: this module uses the KS-97 module which is responsible for remote communication. Through configuring for KS-97, it can connect to the cloud server according to the IP address and port number set before via TCP/IP protocol. Data remote communication is realized by it. This module mainly realizes the function of data forwarding between control module and cloud server.

Control module realizes aggregation and processing of data. The signal source can be controlled by two ways: one is through external buttons; the other is through the GPRS module for remote control. The touch screen is responsible for the display of the data. Current output module is responsible for injecting signal to the fault phase. Working principle is shown in Figure 2. 


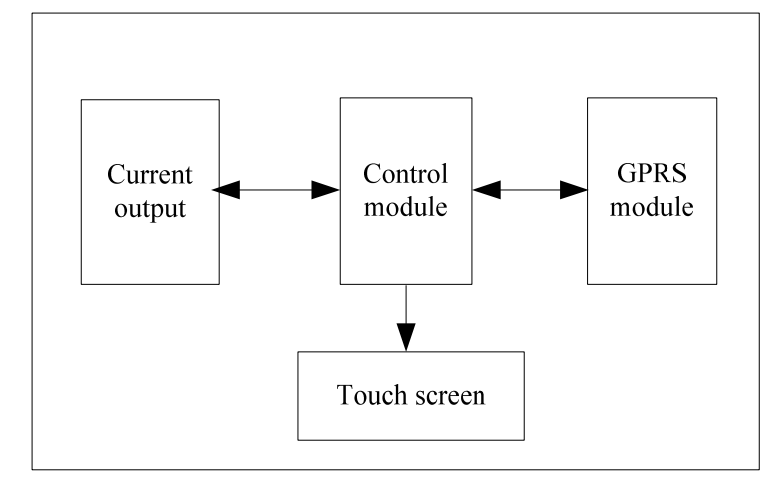

Fig. 2 The working principle diagram of the signal source

Introduction of Handheld Detector and Fault Location Method. Detector inducts and receives AC signal on the line by coil, then the signal will be processed by filter and amplifying circuit.After processing for the signal, the signal will be sent to the control module. After analysis and calculation, control module will sends data to the screen and display; staff can observe the information of signal such as waveform, frequency and current. The principle of detector is shown in Figure 3.

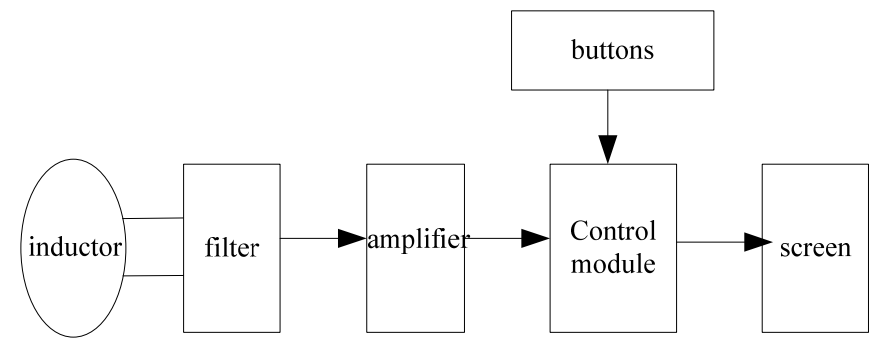

Fig. 3 The principle diagram of detector

When the single-phase-earth fault occurs in distribution network, power supply of lines in fault area will be stopped. After signal source injects signal to the fault phase, current will flow into the earth through the fault point along the line. At the fault point, current exists in the line that near signal source, there is no current in the line that is away from signal source. So the fault point is the cut-off point in the line. According to this obvious characteristic, the fault point is easy to be found by finding the cut-off point, what is the core idea of this fault location method. In addition, in the process of patrol with handheld detector, the work condition of signal source should be real-time monitored by mobile phone client. To achieve the right conclusion, the results from the detector should be analyzed with the help of real-time monitor data from the mobile phone. The injected signal also can be adjusted according to need, to detect the signal more easily, what is the important guarantee of locating the fault.

Stuff with handheld detector uses binary search to search the fault point. In theory, each point on the line is detected will reduce half of the fault area. Generally, the fault point will be found in four times. The position of the detector is shown in Figure 4.

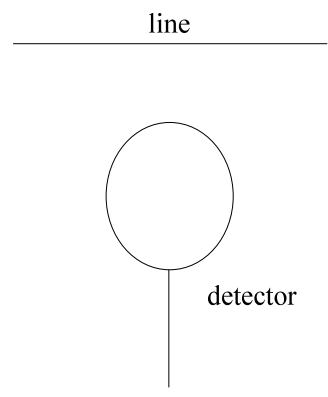

Fig. 4 The position of detector

After signal source injects signal to the fault phase, staff with handheld detector detects the fault line takes the signal source for center. The middle position of line should be detected first. If the detected point has current, the fault point is in downstream, where the line is far away from the 
signal source; if the detected point without current, the fault point is near the signal source.The staff should use binary search to detect the rest of line until the fault point is found.

\section{Design of Mobile Client and Cloud Server}

Design of Mobile Client. This system monitors signal source by mobile application based on Android operating system. Mobile client uses JAVA language to write program. By installing eclipse, ADT and Android SDK programs or components, integrated development of Android applications is realized.

Communication mode uses $\mathrm{C} / \mathrm{S}$ (Client/Server) mode. In this mode, mobile client and server are installed application for communication. The advantages of this mode are convenient for data processing, fast read-write speed, convenient for data real-time transmission. However, the workload of phone is large. Due to this system is of simple function and do not need large workload, $\mathrm{C} / \mathrm{S}$ mode is adopt.

Network communication uses socket programming and TCP/IP protocol, A socket can be connected by an IP address and a port number ${ }^{[7]}$. After the connection is established through the IP address and port number, data transmission can be realized through TCP/IP protocol, then the server can acquire the data of signal source and send control commands to signal source.

The client's main interface is shown in Figure 5. The interface uses RelativeLayout to make it convenient to adjust and manage the individual components. OnClickListener will be triggered if "connect to server" button is clicked. Program will call Onclick() method, then starts socket thread. The connection between mobile phone and cloud server is established through IP and port number, and program will get DataInputStream() and DataOutputStream() methods. Information such as current and frequency of injected signal can be obtained through DataInputStream() method, and the information will be displayed in the TextView. The initial data of TextView is $150 \mathrm{~mA}, 60 \mathrm{~Hz}$, and it will be changed after receiving data. If " $+10 \mathrm{~mA}^{\prime}$ or "-10mA" button is clicked, program will send corresponding control command with GPRS address of signal source to the cloud server by DataOutputStream() method. The cloud server will forward the command to GPRS module of signal source. After receiving the command, signal source will change output signal as the command.

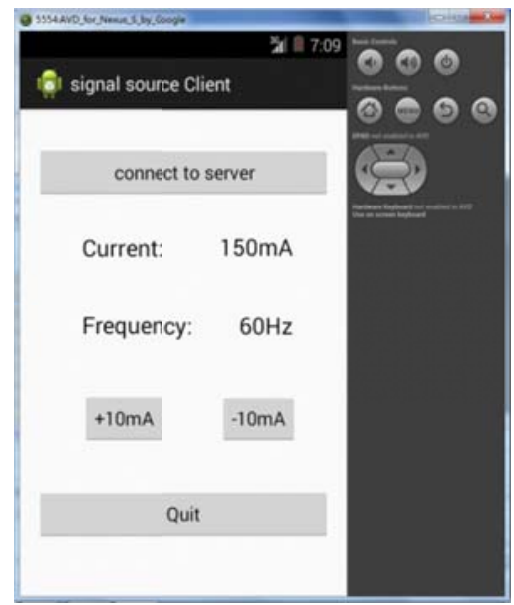

Fig. 5 The client's main interface

Introduction of Cloud Server. Because signal source and mobile client don't have a fixed IP, they cannot transmit data to each other directly via TCP/IP protocol. Cloud server is designed to realize data forwarding. Remote connection and data communication between signal source and mobile client are realized by cloud server forwards upload data of signal source GPRS module and commands of mobile client.

Cloud server overall design uses the service of message bus, what forms a smooth real-time control work mode. In theory, the message bus support infinite client (mobile client and GPRS), and in this form, all events are abstract to message. Message with unified format is convenient for transmission, what reduces the coupling of events and listeners. 
Cloud server designs and configures mapping relation, to implement that any GPRS module can be controlled by any mobile client, what is convenient for remote communication of multiple signal sources and mobile clients later. After client connects to the cloud server, it must send an ID on behalf of itself to the server at first. After the success of the validation, the client was marked, and mapping configuration is read by the server. Then the cloud server will enter work condition. Mobile client commands will be forwarded to the corresponding target signal source according to the mapping relation automatically.

\section{Conclusion}

In the process of fault location with injection scheme, signal source is controlled by mobile to control injected energy remotely. Besides, the injected signal is real-time monitored. This method solves the problem of signal detection for long lines or ultra-high towers and improves the success rate and accuracy of fault location.

Through the combination of cloud server, GPRS module and mobile application, signal source can be controlled remotely, what overcomes the disadvantage of original signal source can't be controlled after open. Staff with a handheld detector can real-time monitor signal source remotely by the mobile client, to grasp the information of injected signal accurately. It is easier to get correct conclusion by the help of observation that detected signal compared to mobile phone, and locate the fault.

\section{Acknowledgments}

This work was financially supported by the Science and Technology Program of State Grid Hebei Electric Power Company (No.TP15-4-KXXM).

\section{References}

[1]. GUO Qingtao, WU Tian, Survey of the methods to select fault line in neutral point ineffectively grounded power system, Power System Protection and Control. 38 (2010) No.2, 146-152.

[2]. Dobakhshari A S, Ranjbar A M, A Wide-area scheme for power system fault location incorporating bad data detection, Power Delivery IEEE Transactions on. 30 (2015) No.2, 800-808.

[3]. SUN Guoqiang, WEI Zhinong, TANG Lifeng, et al, Pareto evolutionary algorithm for multi-objective fault location of distribution network, Electric Power Automation Equipment. 32 (2012) No.5, 57-61+73.

[4]. SI Dongmei, QI Zheng, QIAN Min, et al, Study and realization on fault location about $10 \mathrm{kV}$ distribution network, Power System Protection and Control. 36 (2008) No.18, 24-28.

[5]. GAO Mengyou, XU Bingyin, ZHANG Xinhui, Fault location based on fault current amplitude comparison for active distribution network, Electric Power Automation Equipment. 35 (2015) No.7, 21-25.

[6]. ZHENG Tianwen, XIAO Xianyong, ZHANG Wenhai, el al, Fault location considering nonlinear profile of bus voltage sag for distribution system, Electric Power Automation Equipment. 32 (2012) No.11, 115-120.

[7]. DAI Xin, Research TCP/IP network communication programming on Java Socket, Computer Knowledge and Technology. 9 (2013) No.35, 8116-8117+8153. 